\title{
Comprensión y socialización del concepto género en estudiantes de educación media superior de Pachuca México*
}

\author{
Understanding and Socialization of the Gender Concept \\ in Higher Secondary Education students from Pachuca Mexico \\ Compreensão e socialização do conceito gênero \\ em Estudantes de educação média superior de Pachuca México
}

Recibido el 18 de marzo de 2015. Aceptado el 26 de mayo de 2015

Azul Kikey Castelli Olvera**
México
Rosa María Valles Ruiz***
México

Para citar este artículo:

Castelli Olvera, Azul Kikey,

Valles Ruiz, Rosa María (2015).

Comprensión y socialización del concepto género en estudiantes de educación media superior de Pachuca México. Ánfora, 22 (38),

17-38. Universidad

Autónoma de Manizales. ISSN

0121-6538

\section{Resumen}

Objetivo: comprender cómo entienden y socializan el concepto género, y cómo éste regula las relaciones entre las y los jóvenes estudiantes de nivel medio superior de Pachuca, Hidalgo (México). Metodología: se trabajó con un total de 44 estudiantes de nivel medio superior, divididos en cuatro grupos focales de acuerdo a su plantel de procedencia: Centro Hidalguense de Estudios

\footnotetext{
* Este artículo se deriva del proyecto de investigación en proceso Publicidad social y violencia de género, vinculado con las líneas de investigación que trabajan las autoras de Género y Análisis de Discurso, en la Universidad Autónoma del Estado de Hidalgo (México), institución a la que están adscritas. El proyecto se inició en 2014 y se encuentra en proceso. Está financiado por el Programa Integral de Fortalecimiento Institucional (PIFI) 2015 a cargo de la Licenciatura en Ciencias de la Comunicación de la Universidad Autónoma del Estado de Hidalgo.

** Doctora en Ciencias Sociales. Profesora e investigadora de la Universidad Autónoma del Estado de Hidalgo.Correo electrónico: sakuntala83@yahoo.com.mx

** Doctora en Ciencias Políticas y Sociales. Profesora e investigadora de la Universidad Autónoma del Estado de Hidalgo.Correo electrónico: vallezcurdia@gmail.com y mvalles@uaeh.edu.mx
} 
Superiores, Dr. Alberto Zoebisch, Preparatoria número 1 y Preparatoria número 3 de la Universidad Autónoma del Estado de Hidalgo. A partir del discurso recuperado, se crearon dos categorías de análisis: 1) Los géneros del género; y 2) Igualdades desiguales. Resultados: se detectó que las y los estudiantes entrevistados asocian el concepto género a las características biológicas sexuales de hombres y mujeres. Existe una reiteración de roles tradicionales pese a que en el discurso sostienen que en la actualidad hombres y mujeres son iguales. Conclusiones: en general se observa que aunque empieza a desarrollarse una conciencia de igualdad género entre las y los jóvenes de preparatoria en la Ciudad de Pachuca, Estado de Hidalgo (México) existe aún un discurso que reitera por una parte, los cánones tradicionales, y por el otro, un deber ser de equidad. Se asume la igualdad como lo políticamente correcto, pero al mismo tiempo se reiteran y justifican los roles tradicionales de género.

Palabras clave: Género, Socialización, Jóvenes, Identidades, Roles

\section{Abstract}

Objective: to understand how higher secondary education students comprehend and socialize the concept of gender and how it regulates the relationships among them in Pachuca, Hidalgo (Mexico). Methodology: there was a total of 44 senior high students, divided into four focus groups according to their school: Centro Hidalguense de Estudios Superiores, Dr. Alberto Zoebisch, Preparatoria número 1 and Preparatoria número 3 of the Universidad Autónoma del Estado de Hidalgo. Two analysis categories were created from discourse recovered: 1) genders of gender; and 2) Unequal equalities. Results: the students interviewed associate the concept of gender to sexual biological characteristics of men and women. There is a reiteration of traditional roles although in their discourse, they argue that today men and women are equal. Conclusions: in general, although awareness of equal gender begins to develop among young people of high school in the city of Pachuca, Hidalgo (Mexico), there is still a discourse that reiterates traditional canons, but also claims equity. Equality is assumed as politically correct, but at the same time traditional gender roles are justified and reiterated.

Keywords: Gender, Socialization, Youth, Identities, Roles. 


\section{Resumo}

Objetivo: compreender o jeito como eles entendem e socializam o conceito gênero, e como este regula as relações entre os estudantes jovens dos dois sexos de nível médio superior de Pachuca, Hidalgo (México). Metodologia: trabalhou-se com um total de 44 estudantes de nível médio superior, divididos em quatro grupos focais de acordo a seu plantel de procedência: Centro Hidalguense de Estudos Superiores, Dr. Alberto Zoebisch, Colégio número 1 e Colégio número 3 da Universidade Autónoma do Estado de Hidalgo. A partir do discurso recuperado, criaram-se duas categorias de análise: 1) Os gêneros do gênero; e 2) Igualdades desiguais. Resultados: detectou-se que os estudantes entrevistados associam o conceito gênero às características biológicas sexuais de homens e mulheres. Existe uma reiteração de papeis tradicionais embora no discurso sustentam que na atualidade homens e mulheres são iguais. Conclusões: em geral nota-se que, embora começa a se-desenvolver uma consciência de igualdade gênero entre os jovens de colégio na Cidade de Pachuca, Estado de Hidalgo (México) existe ainda um discurso que reitera por uma parte, os cânones tradicionais, e pelo outro, um dever de equidade. Assume-se a igualdade como o politicamente correto, mas ao mesmo tempo reiteram- se e justificam os papeis tradicionais de gênero.

Palavras-chave: Gênero, Socialização, Jovens, Identidades, Papeis. 


\title{
Introducción
}

El presente trabajo, se origina de una investigación más extensa sobre publicidad social y violencia de género que pretende brindar parámetros para el desarrollo de campañas sociales con el fin de prevenir y atender la violencia de género en jóvenes estudiantes de nivel medio superior ${ }^{1}$, desde el enfoque de la perspectiva de género y los estudios de recepción. Durante la revisión documental y en línea para el desarrollo del estado del arte del estudio mencionado, no se detectaron, por lo menos disponibles para la consulta, análisis sobre campañas de publicidad social para prevenir y atender la violencia de género en México² o estudios de seguimiento y evaluación de las mismas. De ahí la necesidad de conocer ¿cómo se está desarrollando este tipo de publicidad en México, qué tipo de mensajes se están transmitiendo, se rompe con estereotipos o se reiteran, el uso que se le da a las campañas es social o partidista?

En segunda instancia, del acercamiento a los receptores surgen las preguntas: ¿Cómo entienden y socializan ${ }^{3}$ el género los y las estudiantes de preparatoria en

\begin{abstract}
1. Se optó por trabajar con jóvenes debido a que la Encuesta Nacional de Relaciones en los Hogares tanto en 2006 como en 2011 apunta a que las edades en las que se están presentando los mayores índices de violencia se encuentran para 2006, entre los 15 y 29 de años (45.3\%), y para 2011 entre los 15 y 24 años (29.9\%) y de los 25 a los 34 años (30.8\%). Se eligió trabajar con el grupo de menor edad puesto que Philip Kotler apunta que el cambio de actitudes y hábitos es más frecuente en población joven (Kotler, 1992, p. 33). Aunado a lo anterior, siguiendo la opinión de Ángel Hernando Gómez se considera que el inicio de la violencia de género se da cada vez más en edades tempranas (Gómez, 2007, p. 326) Dentro de la diversidad de grupos jóvenes se eligió por realizar el trabajo de campo con estudiantes de nivel medio superior, en consideración a los siguientes aspectos: 1. Viabilidad y acceso a los grupos; 2. Se partió del supuesto de que este grupo tiene un mayor acceso a medios de comunicación por lo tanto, existían mayores probabilidades de que la publicidad social analizada haya sido consumida y que contaran con mayor información sobre violencia de género puesto que además de lo visto en medios de comunicación, el Instituto Hidalguense de las Mujeres ha implementado cursos sobre género y violencia de género, lo que implica que la población seleccionada contaba con algún nivel de sensibilización en esta materia (Instituto Hidalguense de las Mujeres, 2014, p.1); 3. Siguiendo a Graciela Castro (Castro, 2005, p.11) se considera que el espacio educativo es esencial en el desarrollo cultural, pues las escuelas forman parte de las consideradas instituciones dominantes que contribuyen en la adquisición y desarrollo de identidades sociales y son espacios apropiados para la creación, desarrollo y difusión de conocimiento.
\end{abstract}

2. Aunque este tipo de publicidad, ha sido utilizada por asociaciones civiles e instituciones de gobierno en la lucha contra la violencia de género, el uso de este tipo de herramienta mediática para prevención de problemáticas sociales se registra desde 1936, por lo que resulta interesante constatar que sobre el tema existen pocos registros, tal es el caso del trabajo realizado por Griselda Lizcano Álvarez y Liliana Andrea Sánchez Islas, - La publicidad social en México: recuento de 50 años-; la Memoria del Departamento de Prensa y Publicidad y el artículo de Tania Celina Ruiz Ojeda, - La DAPP y el cine como uno de los constructores de la nación mexicana-. Documentos que aportan información histórica sobre el desarrollo de la publicidad social en México, pero no sobre el análisis o evaluación de las campañas implementadas.

3. En el sentido tradicional (y común) de la expresión se dice que un individuo se socializa cuando adquiere una lengua, una serie de normas y valores, va conformando una personalidad. En otras palabras el individuo 
México? ¿Siguen roles tradicionales? ¿Se puede advertir una tendencia a papeles de mayor equidad? ¿Qué elementos están mediando su comprensión y vivencia del género?, para evaluar y proponer alguna modificación o ajuste se requiere la información que arrojan el análisis y estudio de recepción, para plantear discursos ${ }^{4}$ que apelen a los receptores desde sus propios espacios y vivencias, en este caso con respecto al género, la equidad, la violencia y los discursos emitidos por los medios. Para este artículo se retoma sólo lo concerniente a las categorías que ayudan a comprender cómo entienden y socializan el género las y los jóvenes; es decir, cómo se construye la otredad genérica, en este grupo de población.

Se parte de la idea general que distingue el sexo de género; el primero se refiere al hecho biológico de una especie que se reproduce a través de la diferenciación sexual, mientras el segundo guarda relación con los significados que cada sociedad le atribuye a tal hecho. Teresita de Barbieri, citada por Gomáriz (1982) apunta: "Así los sistemas de género son los conjuntos de prácticas, símbolos, representaciones, normas y valores sociales que las sociedades elaboran a partir de la diferencia sexual anátomo- fisiológica y que dan sentido, en general, a las relaciones entre personas sexuadas" (p. 84).

Los estudios de género, tal como lo precisa Inés Alberdi (1999, pp. 9-21), se preocupan por estudiar las relaciones entre hombres y mujeres. El origen de estos estudios es sin duda, el movimiento feminista que se conformó como tal, enarbolando la bandera de la lucha por los derechos de las mujeres en los años setenta del siglo XX, y cuyos antecedentes se ubican en la lucha de las mujeres por su derecho al sufragio en el siglo XIX, e incluso podrían trazarse hasta la Ilustración y la Revolución Francesa.

La periodización antes mencionada permite ubicar la discusión sobre las mujeres y sus derechos en diversos momentos de la historia y sobre todo de la investigación social, visibilizando así, que la preocupación por las mujeres y grupos no hegemónicos ha sido una preocupación por las y los otros, los distintos, desde una postura hegemónica que ha marcado pautas de diferenciación que derivan en exclusión, discriminación y violencia. Por lo anterior, en los

construye una subjetividad, una conciencia práctica y un conjunto de capacidades reflexivas, en la medida en que mantiene relaciones con el medio ambiente natural y social en el que vive (Tenti, 2002)

4. Se refiere al uso pragmático de la lengua sea de modo escrito o verbal. En este uso pragmático el emisor se enuncia a sí mismo a través de su discurso, entendiendo que el sentido se forma en la palabra, la enunciación propone el estudio no del texto, sino del enunciado, suponiendo éste como el ejercicio práctico del uso de la lengua donde quien enuncia se apropia de los signos de ésta para ubicarse con respecto a quien escucha (Benveniste, 1977/1999, p. 83) 
años setenta, la lucha feminista emprendió una campaña política, económica y social para visibilizar a las mujeres y denunciar la posición desigual que éstas ocupaban en todas las sociedades. De esta manera, para los ochentas, cuando el movimiento feminista se instaló en la academia, se retomó el término propuesto por John Money para referirse a los roles culturales que indican lo que es ser hombre y mujer para incluir los estudios sobre las mujeres en el ámbito de la investigación, sin utilizar la palabra mujer:

"Género" parece ajustarse a la terminología científica de las ciencias sociales y se desmarca así de la (supuestamente estridente) política del feminismo. En esta acepción, "género" no comporta una declaración necesaria de desigualdad o de poder, ni nombra al bando (hasta entonces invisible) oprimido (Scott, citado por Lamas, 1996, p. 270).

La visión de lo estudios de género, acota Scott (1996), como estudios referentes a únicamente las mujeres, fue superada y dio paso a una interpretación que incluye necesariamente a los hombres, entendiendo que uno implica al otro. Este uso insiste en que el mundo de las mujeres es parte del mundo de los hombres, creado en él y por él. Rubin (1975) propone el sistema sexo/género, para dar cuenta del conjunto de disposiciones por el que una sociedad transforma la sexualidad biológica en productos de la actividad humana, y en el cual se satisfacen esas necesidades humanas transformadas.

El sistema sexo/género explica las relaciones entre hombres y mujeres y encuentra su soporte teórico en el psicoanálisis y en el marxismo; dos teorías que demarcan la otredad; la primera construye la otredad dentro del mismo sujeto que estudia: en el inconsciente, la segunda, al hablar de burguesía y proletariado. Scott (1996) concuerda con Gayle Rubin (Scott, 1996, p. 286)en diversos aspectos de sus propuestas pero además de ver el género como relaciones de parentesco lo identifica como una forma primaria de relaciones significantes de poder.

Para este trabajo se retoma la propuesta de Joan Scott y Gayle Rubin, entendiendo que el género es una construcción que implica relaciones de poder, donde confluyen mitos, símbolos, normativas, instituciones, relaciones de parentesco e identidad. 


\section{Metodología}

Este trabajo está inscrito en la línea de la metodología cualitativa que reconoce la validez a la expresión de los sujetos participantes en vinculación con un tema cuya especificidad requiere su intervención, en el entendido de que la interpretación de los datos del investigador "no es simplemente un acto cognoscitivo individual, sino además una práctica social y política; como principios centrales de una filosofía interpretativa" (Arias y Giraldo, 2011, p. 501). En este sentido, los grupos focales o focus groups son una técnica de investigación cualitativa que utiliza la entrevista grupal para maximizar o agilizar la recolección de datos, los grupos focales buscan la interacción grupal en torno a diversas preguntas que el moderador va planteando poco a poco. A través de esta técnica se estimula la participación de todo el grupo de trabajo. (Kitzinger, 1995, p. 2).

En cuanto al número de participantes, la cifra varía de acuerdo con el autor. En este caso se retomó la propuesta de Richard Krueger (1994, pp. 20-24), quien sugiere que los grupos focales se integren por un mínimo de cuatro personas y un máximo de doce. Krueger (1994) sostiene que la razón de moverse dentro del rango propuesto se debe a que un grupo focal demasiado grande tiende a subdividirse y es más difícil de manejar, mientras que un grupo pequeño, empobrece la discusión.

Para la selección de las y los participantes que integran los grupos focales se recurrió a un muestreo estratificado (Munch y Ángeles, 2006, pp. 106-107). En este caso se conformaron dos subgrupos: uno con escuelas de educación pública y el otro con escuelas privadas; ambos subgrupos pertenecen al nivel educativo medio superior. Posteriormente, se procedió a jerarquizar las escuelas, considerando la matrícula se organizaron de mayor a menor, se optó por seleccionar las dos que presentan mayor número de estudiantes (dos privadas y dos públicas), los grupos se integraron con dos estudiantes de cada nivel, uno del sexo femenino y otro masculino, dando como total 12 integrantes, 6 mujeres y 6 hombres.

Las instituciones educativas seleccionadas para la realización de los grupos focales en el caso de las escuelas públicas fueron Escuela Preparatoria Núm. Uno y Escuela Preparatoria Núm. 3, pertenecientes a la Universidad Autónoma del Estado de Hidalgo. Para las instituciones privadas se trabajó con la escuela "Dr. Alberto Zoebisch Sánchez” y el Instituto Tecnológico de Monterrey, Campus Hidalgo. 
Se trabajó con un total de 44 estudiantes de nivel medio superior, divididos en cuatro grupos focales de acuerdo con su plantel de procedencia: Centro Hidalguense de Estudios Superiores ${ }^{5}$, Dr. Alberto Zoebisch, Preparatoria Número 1 y Preparatoria Número 3.

Figura 1. Escuelas participantes en grupos focales y número de alumnos

\begin{tabular}{|c|c|}
\hline Escuela de procedencia & $\begin{array}{c}\text { Número de alumnas y alumnos que } \\
\text { participaron }\end{array}$ \\
\hline Cenhies & 12 (6 mujeres y 6 hombres $)$ \\
\hline Dr. Alberto Zoebisch & 10 (5 mujeres y cinco hombres) \\
\hline Preparatoria núm. 1 & 12 (6 mujeres y 6 hombres) \\
\hline Preparatoria núm. 3 & 10 (5 mujeres y cinco hombres) \\
\hline Total general & 44 \\
\hline
\end{tabular}

Fuente: elaboración propia

Con base en la lectura, sistematización, selección y categorización del material transcrito resultante de los grupos focales y considerando los temas ejes abordados en la guía de entrevista, se procedió a ubicar las categorías de análisis pertinentes. Se integraron dos, una de ellas, la más extensa dividida en subcategorías, derivadas de cada uno de los cuatro grupos que se aplicaron, lo que permitió ubicar testimonios coincidentes. A partir de este momento se utilizarán los siguientes códigos cuando se refiera en el texto a las y los participantes de estos grupos:

Figura 2. Referencias y códigos de los grupos focales

\begin{tabular}{|c|c|}
\hline Referencia & Código \\
\hline Participante Grupo Focal 1 & PGF1 \\
\hline Participante Grupo Focal 2 & PGF2 \\
\hline Participante Grupo Focal 3 & PGF3 \\
\hline Participante Grupo Focal 4 & PGF4 \\
\hline
\end{tabular}

Fuente: elaboración propia

\footnotetext{
5. Si bien en el apartado que detalla la selección de las escuelas en las que se llevarían a cabo los grupos focales, hay que aclarar que las dos escuelas privadas seleccionadas fueron: Dr. Alberto Zoebisch y la preparatoria del Tecnológico de Monterrey, Campus Hidalgo, el trabajo de campo en éste último fue imposible debido a que el ciclo escolar había concluido y no había grupos disponibles en el periodo planteado para recolección de campo.
} 
Figura 2. Categorías y significados

\begin{tabular}{|c|c|}
\hline Categoría & Significado \\
\hline Los géneros del género & $\begin{array}{l}\text { Integra los distintos significados que las y los jóvenes le } \\
\text { asignan al concepto género. }\end{array}$ \\
\hline Igualdades desiguales & $\begin{array}{l}\text { Comprende las situaciones específicas en las que las y los } \\
\text { jóvenes empiezan a identificar diferencias en el trato o en la } \\
\text { valoración del comportamiento dependiendo el sexo de la } \\
\text { persona. Esta categoría se subdivide en cuatro subcategorías: } \\
\text { 1. Los ritmos del reloj, también marcan el género; } 2 \text {. Las } \\
\text { máscaras de la violencia en el amor: "somos pareja pero no } \\
\text { somos uno"; 3. La violencia normalizada en el espacio público: } \\
\text { "todo el personal de limpieza son mujeres"; 4. Emotividades } \\
\text { reprimidas. }\end{array}$ \\
\hline
\end{tabular}

Fuente: elaboración propia

\section{Resultados}

En el presente apartado se presentan los resultados del análisis de los datos obtenidos en los grupos focales trabajados con jóvenes estudiantes de preparatoria. Éstos se integran, como se explicó en el apartado de Metodología, en las dos categorías ya mencionadas: Los géneros del género e Igualdades desiguales, ésta última dividida en cuatro subcategorías que se irán presentando en el orden siguiente: 1. Los ritmos del reloj, también marcan el género; 2. Las máscaras de la violencia en el amor: somos pareja pero no somos uno; 3. La violencia normalizada en el espacio público: todo el personal de limpieza son mujeres; 4. Emotividades reprimidas. Para el análisis se transcriben las opiniones y comentarios que las y los jóvenes emitieron en torno al tema y de manera intercalada se van interpretando estos datos, con perspectiva de género.

\section{Los géneros del género}

En su libro El género la construcción cultural de la diferencia sexual, Martha Lamas (1996, p. 327) que la determinación de utilizar la palabra anglosajona gender para sustituir el término estudios de mujeres implicó cierta complicación para los hablantes de lengua hispana. El término gender, género en español, tiene múltiples definiciones, lo que hace el término polisémico, aunado a lo anterior, en inglés gender cuenta con una definición relacionada de manera directa con los sexos, mientras que en español se carece de esa relación inherente entre palabra y concepto. Casi treinta años después, pese al uso reiterado de la palabra género 
tanto en lo político como en lo publicitario la confusión sigue vigente entre la población. En este caso, entre los jóvenes, se entiende género como sinónimo de la palabra sexo:

PARTICIPANTE (Grupo Focal 1 (Hombre: 16 años): Sí, yo creo que sería como una adaptación, anteriormente se decía sexo y ahora no, se utiliza género. PARTICIPANTE (Grupo Focal 2 (Hombre: 17 años): Pues, en lo general cuando habla de género, lo hacen para diferenciar entre algunos casos, por ejemplo en algunos temas, por ejemplo se ha usado tanto para hablar de la diferencia sexo...

PARTICIPANTE (Grupo 3 (Hombre: 15 años): El aparato reproductor.

Como se observa en los comentarios anteriores el concepto género no es entendido como un término que permite, precisamente, diferenciar entre las características sexuales determinadas de manera biológica y la construcción cultural que implica el deber ser mujer o el deber ser hombre sino como un sinónimo de sexo y por tanto de aspectos biológicos.

Pese a la confusión se podría decir que hay cierto avance si se considera que por lo menos los jóvenes empiezan a relacionar la palabra género con hombres y mujeres. Sin embargo, la situación sigue siendo complicada pues la polisemia de la palabra impide concretar significados. De este modo aunque resultó que de entrada lo más recurrente fue la sinonimia entre sexo y género, las y los jóvenes también identificaron otras connotaciones:

PGF1 (Hombre: 15 años): De repente es como clasificación, género de música. PGF2 (Hombre: 16 años): (...) se usa para muchos casos, también se puede hablar de por ejemplo de mercadotecnia.

PGF4 (Mujer: 18 años): ... en el caso de la música.

PGF4 (Hombre 17 años): Yo lo he escuchado en las clasificaciones, género de persona, como dice, de música, hay algunos tipos...

Como se observa las definiciones son múltiples y en este caso se le considera como sinónimo de la palabra tipo, por ello se orienta hacia la clasificación: tipo de música, de películas, en general, como lo comenta una de las participantes del grupo focal, la palabra género para los jóvenes entrevistados se puede aplicar a cualquier conjunto de cosas que compartan características similares entre sí.

Únicamente un participante del grupo 2 relacionó el concepto género como una construcción que va más allá de lo físico, si bien no alcanzó a explicar completamente la idea, si vislumbró con su comentario que entendía que el género a diferencia del sexo no está determinado por aspectos biológicos: 
PGF2 (Hombre: 17 años): Que a diferencia de la palabra sexo, es que por ejemplo, si uno es del sexo masculino no puede cambiar ahora sí que... el aparato reproductor, pero uno puede decidir que es mujer.

El comentario fue aislado y como el grupo no demostró consenso, el joven prefirió adherirse a la concepción general que fue considerar género como sinónimo de sexo. Ahora bien, en un ejercicio más con los grupos se cerró el contexto para tratar de anclar el concepto género en el uso práctico, así que se les preguntó qué entendían por equidad de género, en este caso la respuesta fue similar en los cuatro grupos y hubo consenso y como lo muestran las matrices de comunicación no verbal de cada grupo.

PGF1 (Hombre: 16 años): Es como la igualdad de hombres y mujeres.

PGF2 (Mujer: 17 años): Es que tiene que haber lo mismo para todos, sean distintos o no.

PGF3 (Hombre: 15 años): Que aunque seamos hombres y mujeres, pues tenemos... las mismas capacidades, equivalentes.

PGF4 (Mujer: 16 años): Con los derechos y obligaciones.

Si bien las y los jóvenes identifican a qué se refiere la palabra género con respecto a la equidad, orientan el sentido al concepto legal de acceso a derechos y obligaciones, por lo que pese a que entienden a qué se refiere la equidad de género consideran que ésta se queda en papel y no se lleva a cabo:

PGF2 (Hombre: 17 años): No las tenemos, pero esa es como la idea de equidad que se supone que tenemos que tener como que las mismas oportunidades.

PGF4 (Mujer: 17 años): No lo llevamos a cabo muchas veces.

Lo anterior se debe, al decir de los participantes del grupo 1 y 4, a la permanencia de ideas radicales como el machismo y el feminismo. Cabe mencionar que identifican el feminismo no como una corriente teórica o un movimiento político sino como radical o hembrista equiparable al machismo:

PGF1 (Hombre: 18 años): Yo creo que igual por esa parte podría no terminarse la violencia, porque podríamos irnos al otro extremo, por feminismo total, y también por eso habría muchos problemas, desigualdad también habría, yo digo.

PGF4 (Hombre: 17 años): Por el machismo o cosas así.

PGF4 (Mujer: 15 años): O por el contrario, el feminismo. 
Con los antecedentes que aporta el apartado anterior, se parte de dos conclusiones básicas: existe una confusión en la comprensión del concepto género provocada por la polisemia de la palabra en español, que no se ha podido resolver luego de tres décadas de uso del concepto para referirse a construcciones culturales y relaciones de poder entre los sexos, que es la definición anglosajona de la palabra gender. Si bien al anclar el significado de la palabra género vinculándolo con equidad hay una mayor comprensión del concepto compuesto, pues los jóvenes lo relacionan con igualdad entre hombres y mujeres frente a la ley, mismas oportunidades, etc., se vislumbra que consideran el uso del concepto equidad de género como un término legal o político pero que en la vida cotidiana no se aplica por cuestiones culturales que demarcan diferencias que contribuyen a la desigualdad:

En la esfera política, sin embargo, podemos advertir algunos cambios que a primera vista podrían parecer contradictorios con la situación social antes descrita: la presencia y mayor visibilidad de las mujeres políticas y las referencias constantes por miembros de la clase política al discurso de género. Estos dos fenómenos se desarrollan en un ambiente socio-cultural sexista y misógino, al que debe sumarse la derechización del discurso político que trajo el gobierno de Fox (Rodríguez, 2005, p. 51).

\section{Igualdades/desiguales}

Esta categoría comprende las situaciones específicas en las que las y los jóvenes empiezan a identificar diferencias en el trato o en la valoración del comportamiento dependiendo el sexo de la persona. Esta diferenciación determinada por el género incide en el control del tiempo, las relaciones de pareja, el modo en que conciben lo femenino y lo masculino, las posibilidades escolares laborales y las emotivas. La categoría se subdivide en cuatro subcategorías que se presentan en el siguiente orden: 1. Los ritmos del reloj, también marcan el género; 2. Las máscaras de la violencia en el amor: "somos pareja pero no somos uno"; 3. La violencia normalizada en el espacio público: "todo el personal de limpieza son mujeres"; 4. Emotividades reprimidas.

\section{Los ritmos del reloj también marcan el género}

Las y los jóvenes identificaron diversos espacios en donde, pese a que reconocen, deberían existir condiciones de igualdad, existen diferencias claras en las reglas sociales y conductuales que la sociedad impone dependiendo el 
género, uno de los primeros elementos que identificaron fue el control del tiempo diferenciado:

PGF1 (Hombre: 17 años): Por ejemplo yo tengo, mi hermana y pues no la van a dejar salir como que a la misma hora que a mí.

PGF2 (Mujer: 16 años): Por ejemplo cuando vas a pedir un permiso o algo, a una fiesta o algo, tu papá te tiene que llevar, en cambio si sale el hermano, no importa.

PGF2 (Hombre: 17 años): Pues, por ejemplo en el caso del deporte, a mí me dicen, no pues vete a jugar, y a mis hermanas les dicen, este, bueno si quieren salir a alguna fiesta, no pues ¿a qué hora llegan?, que vaya aparte tu hermano, y cuando yo voy a una fiesta o algo, no pues ¿a qué hora llegas?, y ya, y si llego más tarde me dicen que ya no llegue tarde, pero a ellas sí las castigan.

PGF3 (Mujer: 18 años): Apenas si nos dan permiso.

Este control del tiempo diferenciado se asocia con el caso de las mujeres a las que se les considera más propensas a un ataque sexual; mientras que en el caso de los hombres consideran que el mayor riesgo que corren es un asalto, entran en juego al parecer ciertos aspectos de control moral que deriva en control del cuerpo femenino vinculado a aspectos sexuales:

PGF3 (Hombre: 18 años): Bueno en cuanto a fiestas yo creo que es por seguridad, porque una niña puede ir solita al autobús, y pueden llegar muchos hombres y la violan o algo, y a un hombre no, sólo le quitan su celular o algo así...

PGF4 (Mujer: 17 años): Ahora sí que le dan más la confianza al hombre porque digamos corre menos... o sea corre los mismos riesgos, pero según los papás corren menos riesgos que las mujeres.

Pese a que las y los entrevistados son jóvenes cuyas edades oscilan entre los 15 y 18 años, el estereotipo de la mujer como sinónimo de frágil o vulnerable persiste y se considera como una razón para el control del tiempo:

PGF4 (Hombre: 17 años): Porque la mujer es más indefensa.

PGF4 (Mujer: 15 años): No, por eso, sí nos dan permiso pero no mucho tiempo, por eso, pero si ya es muy tarde van por nosotros, tengo tres hermanas, entonces es así como... como dicen, creen que somos vulnerables por lo mismo, entonces es como más protección.

De acuerdo con los comentarios expuestos existen reglas más estrictas con respecto al uso del tiempo en el caso de las mujeres, los padres preguntan con quién salen, a dónde y a qué hora llegan, si no se cumple con lo establecido entonces se impone un "castigo", mientras que el caso de los hombres únicamente hay un llamamiento de atención. Así mismo, las explicaciones solicitadas son menores: 
PGF4 (Hombre: 17 años): Si saben que voy a ir con mis amigos, sólo me dicen cuídate, protégete.

El control del tiempo diferenciado se podría relacionar con una reiteración de los espacios en los que tradicionalmente se les ubica a hombres y mujeres, para ellos el ámbito público y para ellas el espacio privado o el hogar. Esto indica que aún existe cierta persistencia de roles tradicionales de género, aunque éstos no sean percibidos de manera consciente por las y los jóvenes, pues justifican el control como una medida de seguridad para las mujeres, lo mismo ocurre en la siguiente subcategoría, tal y como se observa en los comentarios de las y los entrevistados.

\section{Las máscaras de la violencia en el amor: "somos pareja pero no somos uno"}

Otro espacio en donde se identificaron diferencias es el noviazgo, se les plantearon a las y los jóvenes preguntas con respecto a ciertos comportamientos durante el mismo para comprender cómo lo explicaban y si identificaban situaciones de violencia. Uno de los primeros temas abordados fueron los celos, en este caso la mayoría de los participantes comentaron que las relaciones en donde se presentaba acoso o control por parte de la pareja se consideraban como indeseables:

PGF1 (Mujer: 17 años): Yo sí lo interpreto como acoso, porque si estás en una relación, es porque ambas parte tienen que cooperar, pero manteniendo su individualidad y su independencia.

PGF2 (Mujer: 16 años): Ni casados tienen el derecho de... ni como mujer, ni como hombre, de estar atosigando a la otra persona.

Aunque la primera reacción fue de rechazo, en cuanto alguno de los participantes expresó cierta justificación para sentir celos, los grupos reajustaron sus posturas pasando del rechazo a cierto nivel de tolerancia, en el caso del grupo 1 el detonante fue el cometario siguiente:

PGF1 (Hombre: 17 años): Si yo siento que a lo mejor no todo lo que es de celos significa violencia, también este, de un celo a otro celo, un celo normal, pues quiere decir que te quiere ¿no?, hay otros celos que también ya se van a la locura, de que a cada rato ¿dónde andas?, ¿qué estás haciendo?, ¿con quién estás? 
Es decir, se pasó del rechazo a la justificación a partir de "ciertos estándares de normalidad” permitidos en pro del amor, la protección o la atención:

PGF2 (Mujer: 16 años): Es que muchas veces tanto el hombre como la mujer se permite, porque en un principio no te das cuenta, a lo mejor lo ves como algo lindo, como que siempre quiere estar al lado de ti.

PGF2 (Mujer: 17 años): Ajá, como que te cuida, te protege.

PGF1 (Mujer: 17 años): O por ejemplo hay gente que puede muy bien encauzar los celos, hay gente que por ejemplo algún detalle celoso, en lugar de hacerla sentir atacada, le haces sentir que le importas ¿no?, y no estás encauzando muy mal tus celos, ni estás volviendo mala la relación, simplemente como que le estás haciendo saber que te importa pero al grado de no invadir su espacio personal.

Como se observa, hubo compañeros que detonaron el cambio de postura en torno a los celos, pero después de expresar su opinión en el tema mantuvieron un nivel muy bajo de participación en el resto de la dinámica de grupo focal. Tal es el caso del grupo 1 donde quien detonó el cambio de postura no participó de nuevo, lo que implica cierto desacuerdo con el grupo y temor a una sanción social, puesto que pese a que se expresó cierta tolerancia a los "celos normales" se mantuvo la idea de necesidad de independencia y respeto:

PGF1 (Mujer: 17 años): es que necesitan darse su espacio, por ejemplo si se ven aquí en la escuela y toda la tarde están mandándose mensajes, siento que es... como que uno termina hartándose del otro ¿no?, invade tu espacio personal, ese tipo de acciones no deben de justificarse como amor.

PGF2 (Mujer: 16 años): De hecho, si tienes una pareja es para compartir, obviamente no todas tus cosas porque uno tiene cosas personales, pero sí compartes con tu pareja, pero hay una donde dices hasta aquí o sea, lo demás ya es muy mío, somos pareja, no somos realmente una sola persona, nunca vas a estar... o sea no sabes si vas a estar con esa persona para toda la vida.

Si bien se observa cierta flexibilización en el modo en que se vive una relación de noviazgo tendiente a la equidad relacionada con el uso del tiempo y la individualidad, así como una tendencia paulatina a la desnaturalización de los celos como muestra de amor.

En el aspecto económico los hombres aún se consideran como responsables de las mujeres y como proveedores, donde la imposibilidad de cumplir con "rituales de cortejo" como el pago de comidas, cafés o la compra de regalos para la novia se considera como una pérdida de la virilidad que implica que si la novia invita al 
novio, las características de dependencia que se le suelen asignar a las mujeres se transfieren al hombre, que es entonces comparado con una mujer:

PGF4 (Mujer:16 años): ...por ejemplo, en una relación, que es el hombre que el que tiene que pedir a la chava que sea la novia, o que él es el que le tiene que dar los regalos o el que le tiene que pagar a la mujer, o sea todavía se tienen esas cosas y en eso pues no hay equidad de género, se tiene mucho de que la mujer es... la que tiene que ser más protegida o la que puede estar ahí haciendo sus berrinches y el hombre es el que tiene que solucionarlo...

PGF4 (Hombre: 17 años): Porque pues quizá si también nos dice la chava, pues no le vas a decir ¿quieres algo?, no sé, pero no le vas a decir la mitad y la mitad ¿no?

PGF4 (Hombre: 17 años): Osea, le dirían al hombre, jay sí!, a ti... qué tú eres la mujer en la relación, no.

La validación de un esquema de masculinidad hegemónico donde el hombre es el responsable de iniciar y guiar las relaciones de noviazgo, donde los celos se admiten como "muestras de amor o interés", se trasluce en el discurso de las y los entrevistados, ya que por un lado dicen que es inadmisible que la pareja o novio controle la relación, y por otro se esperan ciertas pautas de comportamiento masculino que coinciden con roles de género tradicionales. Esto se observa en esta categoría que refiere a un ámbito personal pero se reitera también en el espacio público, por ejemplo en la escuela y el trabajo tal y como se explica en la siguiente subcategoría.

\section{La violencia normalizada en el espacio público: "todo el personal de limpieza son mujeres"}

Las y los jóvenes que participaron en los grupos focales comentaron que en el ámbito escolar y profesional observan que la inclusión de las mujeres en estos espacios es cada vez mayor, sostienen que las oportunidades de estudio y trabajo son iguales para hombres y mujeres, aunque reconocen que existen límites sociales y ejemplos dados reiteran la ubicación de las mujeres en trabajos relacionados con la limpieza o la preparación de alimentos lo que resulta extensivo al "rol tradicional” de la mujer:

PGF2 (Hombre: 16 años): Tenemos un ejemplo aquí, el personal de toda la, la limpieza es mujer, no hay tantos hombres, y pues para mi criterio, pienso que en el trabajo es mejor una mujer que un hombre. 
PGF2 (Mujer: 17 años): Todavía no por completo, pero sí ya hay más aceptación a la mujer en diferentes ramas de trabajo.

Las y los participantes aceptaron que la inclusión exitosa de hombres y mujeres en profesiones que tradicionalmente se asignaban a uno u otro género, es cada más frecuente, aún existe un costo social donde se les etiqueta o excluye por pertenecer a uno u otro sexo:

PGF4 (Hombre: 17 años): ... que la carrera la puede estudiar cualquiera, porque ya hay esa equidad de que cualquiera puede estudiar lo que quiera, puede haber ingenieras o secretarios o cosas así.

PGF4 (Mujer: 17 años): Pero la sociedad es la que lo ve mal ¿no?, por ejemplo un hombre estilista...

PGF4 (Hombre: 16 años): Es gay.

PGF4 (Hombre: 18 años): ... una mujer que trabaja en... taller mecánico o algo así... PGF4 (Hombre: 17 años): Es machorra...es lesbiana o algo así.

Aunada a la sanción social también observan cuestiones de discriminación salarial y laboral vinculadas con violencia de género en el trabajo:

PGF3 (Mujer: 16 años): Igual, no sé, en los trabajos hay veces que a las mujeres les pagan menos, y al hombre le pagan más.

PGF3 (Hombre: 17 años): El problema no es estudiar la carrera, el problema es encontrar trabajo, pues porque siempre habrá esa diferencia, no todos contratan a una mujer.

Con respecto a la participación masculina como encargados del hogar comentaron que si bien, se acepta que las mujeres trabajen en el espacio público, aún resulta controversial el hecho de algunos hombres trabajen en sus casas, pues implica dependencia y cierta pérdida de virilidad:

PGF2 (Hombre: 17 años): Eso de que los hombres se quedan un poco más en casa y no hagan nada y de que la mujer se vaya a trabajar, todavía no está bien visto, eso sí todavía no está bien visto, pero...

PGF2 (Mujer: 17 años): Eso está mal visto para la sociedad, está como de mantenido...

PGF2 (Hombre: 16 años): Zángano

Lo anterior se relaciona con la imagen del hombre como proveedor la cual se conforma desde pequeño, se reitera durante el noviazgo y se mantiene durante las relaciones adultas de pareja. De modo que el no cumplir con el "rol tradicional" 
implica una carencia de poder y autoridad frente a la "mujer proveedora", lo que puede derivar en frustración y agresión del varón hacia su familia como respuesta a la presión social y a la represión emotiva que implica la construcción de la masculinidad tradicional, tal y como se trasluce en el siguiente apartado.

\section{Emotividades reprimidas}

La posibilidad de expresión emotiva es una de las subcategorías donde las y los jóvenes encuentran diferencias más claras, el ser emotivo o llorar está permitido para las mujeres pero no para los varones:

PGF4 (Mujer: 18 años): No es natural que no llores, es natural hasta cierto punto porque que las mujeres son, tienen, digamos más emociones o... pero no por completo así de son las más chillonas, o las que más siente, pero para eso () pero sí somos un poco más sensibles...

PGF2 (Mujer: 17 años): Por ejemplo, mi papá, nunca en mi vida que yo recuerde me ha demostrado como un sentimiento...

Generación tras generación el discurso se modifica pero no el trasfondo. Al respecto uno de los participantes comentó que su abuelo sí habló con él sobre que el llorar no era para hombres:

PGF4 (Hombre: 17 años): a mí sí mi abuelo me llegó a decir así “los niños no lloran”

Por otro lado, en generaciones más recientes el padre no hace alusión a la expresión emotiva pero el trasfondo sigue siendo el mismo, pervive el temor de que los hijos varones presenten características "poco masculinas":

PGF4 (Hombre: 17 años): Nada más sé machito, no me salgas gay.

Las y los jóvenes reconocen que las posibilidades de expresión de unos y otras son aprendidas siendo el principal agente socializador: la familia:

PGF4 (Hombre: 16 años): Yo digo que un poco igual más aprendido...Porque es algo que nos enseñan nuestros papás de que los hombres no deben llorar o algo así.

PGF4 (Mujer: 17 años): Yo siento que esas cosas son aprendidas, porque no necesariamente tiene que ser... bueno, no siento que sea tanto de naturaleza, uno puede cambiar, puede cambiar de la actitud y todo eso. 
Aunque se reconoce que es un comportamiento aprendido, la posibilidad de expresar o no las emociones se ve limitada por la sanción social que se refleja en burla y discriminación:

PGF4 (Hombre: 18 años): Parece niña.

PGF4 (Mujer: 17 años): Sí, cuando alguien... cuando un chico llega a llorar en público, es muy raro que un hombre se acerque a él y le diga ¿estás bien?, o ¿qué te pasó?

PGF4 (Hombre: 17 años): Por lo general siempre dicen que es gay...

Esta construcción de la masculinidad vinculada al poder y al estoicismo deriva como lo menciona Michel Kaufman (1999, p.1) en la imposibilidad masculina de expresar miedo o tristeza si no es a través de la ira o la violencia, lo que implica paternidades poco afectivas y la reiteración de la violencia de género. Kaufman desagrega la construcción de la masculinidad en lo que denomina "Las siete P's de la violencia de los hombres”, en este sentido los comentarios de las y los jóvenes entrevistados corresponderían con la primera p, que establece que para edificar las jerarquías de dominación de los hombres sobre las mujeres y también sobre otros hombres, se utiliza como mecanismo la violencia o la amenaza, el resultado es que los hombres interiorizan la violencia, y en consecuencia no sólo aprenden a utilizarla de manera selectiva sino que también aprenden a transformar una gama de emociones en ira.

\section{Conclusiones}

Aunque las y los jóvenes logran vincular el concepto de género y equidad con igualdad, observan que: esto sólo es a nivel jurídico o parte de un discurso que no coincide con el cómo entienden y socializan el concepto género, objetivo éste del presente artículo ni en cómo el género regula las relaciones entre ellos, ya que las prácticas cotidianas en las que se desenvuelven en su vida diaria, los espacios del género se encuentran bien delimitados, aunque se piense que existe igualdad entre ellas y ellos, al planteárseles espacios específicos identifican diferencias en el control del tiempo, relaciones de noviazgo, posibilidades emotivas y espacios de trabajo, que en algunos casos, las y los jóvenes justifican, en una reiteración de las relaciones de poder entre los sexos, señaladas por Joan Scott y legitimadas a través del discurso de la familia, la escuela y los medios.

A través del análisis se observa distintos tipos de discurso. Por una parte de manera abierta las y los jóvenes comentan que están de acuerdo en que hombres y mujeres son iguales pero legitiman por ejemplo: el control del tiempo femenino 
bajo la justificación de que las mujeres son más frágiles y podrían ser víctimas de un ataque de índole sexual y deben cuidarse más, mientras que a los hombres "sólo los asaltan", esto es reiterado tanto por hombres como por mujeres. Se justifica así un control sobre los cuerpos y los tiempos de las mujeres, que tiene que ver con roles tradicionales donde lo femenino pertenece al espacio privado mientras que lo masculino se mueve en el espacio público. Estos discursos son inconscientes, pues el género se encuentra normalizado, introyectado, por lo que las y los jóvenes no identifican incoherencias entre la igualdad entre hombres y mujeres y los controles diferenciados que en este caso, la familia impone a las y los jóvenes.

Las posibilidades emotivas siguen siendo limitadas por la pertenencia a uno u otro género, a las mujeres se les permite llorar, mientras que los hombres tienen que mostrar fortaleza para evitar comparaciones con las mujeres. Los jóvenes comentaron que en sus familias aún está presente el temor de que sus hijos presenten una orientación sexual diferente a la heterosexual por lo que alientan las conductas que consideran "masculinas" (Kaufman, 1999, p.1).

En general, se observa que aunque empieza a desarrollarse una conciencia de igualdad de $\mid$ género entre las y los jóvenes de preparatoria en la Ciudad de Pachuca, los discursos antes mencionado, se reitera en todos los aspectos tratados, se asume la igualdad como lo políticamente correcto, pero al mismo tiempo se reiteran y justifican los roles tradicionales de género que conciben lo femenino como lo controlable y frágil y lo masculino como libre y fuerte, lo que justifica una serie de mecanismos, muchas veces violentos, como la burla e incluso la agresión verbal o física hacia aquellos o aquellos que no cumplen con los estándares establecidos socialmente.

\section{Referencias}

Alberdi, I. (1999). El significado del género en las ciencias sociales. Política y sociedad, (32), 9-21.Universidad Complutense de Madrid. Recuperado de: http://redalyc.uamex.mx.

Arias, M. M y Giraldo, C. V. (2011). El rigor científico en la investigación cualitativa. Investigación Educativa Enfermería, 29. Colombia, Universidad de Antioquia. 
Benveniste, E. (1977). Problemas de lingüística general II. España: Siglo veintiuno editores.

Castro, G. (2005). Los jóvenes y la vida cotidiana: elementos y significados de su construcción. Espacio Abierto. Cuaderno Venezolano de Sociología, 14(1).

Gomáriz, E. (1992). Los estudios de género y sus fuentes epistemológicas: periodización y perspectivas. Isis internacional, (17).

Gómez, Á. (2007). La prevención de la violencia de género en adolescentes. Una experiencia en el ámbito educativo. España: Universidad de Sevilla.

Kaufman, Michael, Las siete P's de la masculinidad. Recuperado de: http://www. michaelkaufman.com/wp-content/uploads/2009/01/kaufman-las-sieteps-de-la-violencia-de-los-hombres-spanish.pdf.

Kitzinger, J. (1995). Qualitative Research: Introducing focus group, Estados Unidos, BMJ, 1995. Recuperado de: http://www.bmj.com/cgi/ pmidlookup? view $=$ long\&pmid $=763324$.

Kotler, R. E. (1992). Marketing social. Estrategias para cambiar la conducta pública. Madrid, España: Ed. Díaz de santos.

Krueger, R. (1994). Focus group: a principal guide for applied research, Newbury Park CA.

Instituto Hidalguense de las Mujeres (2014). Talleres impartidos en materia de Igualdad entre Mujeres y Hombres, derechos humanos de las mujeres y prevención de la violencia contra las mujeres en el periodo 20122014. Estado de Hidalgo, e Instituciones de Educación Media Superior. Subdirección de género. Instituto Hidalguense de las Mujeres.

Lamas, M. (1996). "Usos, dificultades y posibilidades de la categoría género". En Marta Lamas (Comp.). El género: la construcción cultural de la diferencia sexual. México:

Munch, L. y Ángeles, E. (2006). Métodos y técnicas de investigación, México: Ed. Trillas. 
Rodríguez, T. (2005). Porque no es lo mismo decir gallo que gallina. Discurso político y representaciones de género en la nueva democracia mexicana. Andamios, (3), p.51-75.

Rubin, G. (1996). El tráfico de mujeres: notas sobre la economía política del sexo. En Marta Lamas. El género: la construcción cultural de la diferencia sexual. Nueva Antropología, VIII (30). México: Universidad Nacional Autónoma de México.

Scott, J. (1996). El género una categoría útil para el análisis histórico. En Marta Lamas. El género: la construcción cultural de la diferencia sexual. Nueva Antropología, Vol. VIII, núm. 30, México, Programa Universitario de Estudios de Género.

Tenti, E. (2002). Socialización. En Carlos Altamirano (Ed.). Términos críticos. Diccionario de sociología de la cultura. Buenos Aires, Argentina: Paidós. 